University of Wollongong

Research Online

Faculty of Engineering - Papers (Archive)

Faculty of Engineering and Information

Sciences

June 2004

\title{
A Systematic Study of Radio-induced X-Ray Cavities in Clusters, Groups, and Galaxies
}

L. Birzan

Ohio University, USA

D. A. Rafferty

Ohio University, USA

B. R. McNamara

Ohio University, USA

M. W. Wise

Massachusetts Institute of Technology, USA

P. Nulsen

University of Wollongong

Follow this and additional works at: https://ro.uow.edu.au/engpapers

Part of the Engineering Commons

https://ro.uow.edu.au/engpapers/297

\section{Recommended Citation}

Birzan, L.; Rafferty, D. A.; McNamara, B. R.; Wise, M. W.; and Nulsen, P.: A Systematic Study of Radioinduced X-Ray Cavities in Clusters, Groups, and Galaxies 2004.

https://ro.uow.edu.au/engpapers/297

Research Online is the open access institutional repository for the University of Wollongong. For further information contact the UOW Library: research-pubs@uow.edu.au 


\title{
A SYSTEMATIC STUDY OF RADIO-INDUCED X-RAY CAVITIES IN CLUSTERS, GROUPS, AND GALAXIES
}

\author{
L. Bîrzan, D. A. RAFFerty, AND B. R. McNamara \\ Department of Physics and Astronomy, Ohio University, Clippinger Laboratories, Athens, OH 45701 \\ M. W. WISE \\ Center for Space Research, Building NE80-6015, Massachusetts Institute of Technology, Cambridge, MA 02139 \\ AND \\ P. E. J. NULSEN \\ Harvard-Smithsonian Center for Astrophysics, 60 Garden Street, Cambridge, MA 02138; and \\ School of Engineering Physics, University of Wollongong, Wollongong, NSW 2522, Australia \\ Received 2003 December 12; accepted 2004 February 10
}

\begin{abstract}
We present an analysis of 16 galaxy clusters, one group, and one galaxy drawn from the Chandra Data Archive. These systems possess prominent X-ray surface brightness depressions associated with cavities or bubbles that were created by interactions between powerful radio sources and the surrounding hot gas. The central galaxies in these systems harbor radio sources with luminosities ranging between $\sim 2 \times 10^{38}$ and $7 \times 10^{44} \mathrm{ergs} \mathrm{s}^{-1}$. The cavities have an average radius of $\sim 10 \mathrm{kpc}$, and they lie at an average projected distance of $\sim 20 \mathrm{kpc}$ from the central galaxy. The minimum energy associated with the cavities ranges from $p V \sim 10^{55}$ ergs in galaxies, groups, and poor clusters to $p V \sim 10^{60} \mathrm{ergs}$ in rich clusters. We evaluate the hypothesis that cooling in the hot gas can be quenched by energy injected into the surrounding gas by the rising bubbles. We find that the instantaneous mechanical luminosities required to offset cooling range between $1 p V$ and $20 p V$ per cavity. Nearly half of the systems in this study may have instantaneous mechanical luminosities large enough to balance cooling, at least for a short period of time, if the cavities are filled with a relativistic gas. We find a trend or upper envelope in the distribution of central X-ray luminosity versus instantaneous mechanical luminosity, with the sense that the most powerful cavities are found in the most X-ray-luminous systems. Such a trend would be expected if many of these systems produce bubbles at a rate that scales in proportion to the cooling rate of the surrounding gas. Finally, we use the X-ray cavities to measure the mechanical power of radio sources over six decades of radio luminosity, independently of the radio properties themselves. We find that the ratio of the instantaneous mechanical (kinetic) luminosity to the $1.4 \mathrm{GHz}$ synchrotron luminosity ranges typically between a few and roughly a few thousand for luminous radio sources but can be several thousand for weaker sources. This wide range implies that the $1.4 \mathrm{GHz}$ synchrotron luminosity is an unreliable gauge of the mechanical power of radio sources.
\end{abstract}

Subject headings: galaxies: active — galaxies: clusters: general — X-rays: galaxies — X-rays: galaxies: clusters

\section{INTRODUCTION}

The cooling time of the intracluster gas in the cores of many galaxy clusters is shorter than 1 Gyr. In the absence of heating, a "cooling flow" (Fabian 1994) is established, in which the gas cools below X-ray temperatures and accretes onto the central cluster galaxy, where it accumulates in molecular clouds and forms stars. Chandra images of cooling flow clusters have confirmed the existence of inwardly decreasing temperature gradients and short central cooling times, which are the distinguishing characteristics of a cooling flow. However, moderate-resolution Chandra and $A S C A$ spectra and highresolution XMM-Newton spectra (e.g., Makishima et al. 2001; Peterson et al. 2001; Tamura et al. 2001; Kaastra et al. 2004) do not show the expected signatures of cooling below $2 \mathrm{keV}$, reported to exist in lower resolution data from the Einstein and ROSAT observatories. This discrepancy would be difficult to understand unless the normal signatures of cooling below $2 \mathrm{keV}$ are somehow suppressed, or if cooling is indeed occurring but at rates that are generally factors of 5-10 lower than expected (e.g., Molendi \& Pizzolato 2001; Böhringer et al. 2002; Peterson et al. 2003). Several scenarios have been suggested that may suppress the cooling flux at low energies and yet maintain the large cooling rates. The list includes the effects of differential absorption, efficient mixing, inhomogeneous metallicity distributions (e.g., Fabian et al. 2001). However, these scenarios, as clever as they are, may require fine-tuning and are otherwise difficult to prove observationally. In any case, they leave the question of the repository for most of the cooling gas unanswered.

The more appealing interpretation, which we address in this paper, posits that radiation losses are being balanced, or nearly so, by heating, implying that the large cooling rates of the last decade were indeed overestimated. This suggestion has its own difficulties. Maintaining gas with a cooling time approaching $100 \mathrm{Myr}$ at $\mathrm{keV}$ temperatures almost certainly requires the existence of one or more heating mechanisms operating in a self-regulating feedback loop. One such mechanism, thermal conduction from the hot outer layers of clusters, may be energetically feasible, in some instances (e.g., Tucker \& Rosner 1983; Bertschinger \& Meiksin 1986; Zakamska \& Narayan 2003; Voigt et al. 2002; Voigt \& Fabian 2004). However, it generally requires fine-tuning and can be unstable (Bregman \& David 1988; Soker 2003). Moreover, conduction operating alone at even the Spitzer rate cannot offset radiation losses (Voigt et al. 2002; Wise et al. 2004) in all clusters and is 
TABLE 1

Radio Properties

\begin{tabular}{|c|c|c|c|c|c|c|}
\hline System & $z$ & $\begin{array}{c}\sigma^{\mathrm{a}} \\
\left(\mathrm{km} \mathrm{s}^{-1}\right)\end{array}$ & $\begin{array}{c}S_{1400} \\
(\mathrm{Jy})\end{array}$ & $\alpha^{\mathrm{b}}$ & $\begin{array}{c}P_{1400} \\
\left(10^{24} \mathrm{~W} \mathrm{~Hz}^{-1}\right)\end{array}$ & $\begin{array}{c}L_{\text {radio }} \\
\left(10^{42} \text { ergs s }^{-1}\right)\end{array}$ \\
\hline Cygnus A ............... & 0.056 & $\ldots$ & $1598 \pm 41$ & $0.7(4)$ & $11800 \pm 300$ & $700 \pm 20$ \\
\hline Hydra A ................ & 0.052 & $322 \pm 20(8)$ & $40.8 \pm 1.3$ & $0.92(4,13)$ & $261 \pm 8$ & $19.9 \pm 0.6$ \\
\hline 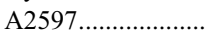 & 0.085 & $224 \pm 19(16)$ & $1.875 \pm 0.056$ & $1.35(11)$ & $35 \pm 1$ & $6.8 \pm 0.2$ \\
\hline MKW 3S................ & 0.045 & $\ldots$ & $115.0 \pm 3.9$ & $2.3(4,13,17)$ & $0.58 \pm 0.02$ & $3.6 \pm 0.1$ \\
\hline A2052 .................... & 0.035 & $253 \pm 12(19)$ & $5.50 \pm 0.21$ & $1.2(1,4,13)$ & $15.7 \pm 0.6$ & $2.08 \pm 0.08$ \\
\hline A133 & 0.060 & $\ldots$ & $0.167 \pm 0.006$ & $1.9(14,15)$ & $1.54 \pm 0.06$ & $1.94 \pm 0.07$ \\
\hline A4059..................... & 0.048 & $296 \pm 49(6)$ & $1.284 \pm 0.043$ & $1.43(13,18,21)$ & $6.96 \pm 0.2$ & $1.73 \pm 0.06$ \\
\hline A2199.................... & 0.030 & $295 \pm 6(7)$ & $3.58 \pm 0.12$ & $1.37(1,4,17)$ & $7.43 \pm 0.2$ & $1.56 \pm 0.05$ \\
\hline Perseus .................... & 0.018 & $246 \pm 10(8)$ & $22.83 \pm 0.68$ & $1.0(10)$ & $16.5 \pm 0.5$ & $1.42 \pm 0.04$ \\
\hline RBS $797 \ldots \ldots \ldots \ldots . . . . .$. & 0.350 & $\ldots$ & $0.0217 \pm 0.0008$ & $\ldots$ & $9.0 \pm 0.3$ & $0.78 \pm 0.03$ \\
\hline A1795 ..................... & 0.063 & $297 \pm 12$ & $0.925 \pm 0.028$ & $0.98(1,4,13)$ & $9.0 \pm 0.3$ & $0.75 \pm 0.02$ \\
\hline M87 ...................... & 0.0042 & $330 \pm 5(2)$ & $138.5 \pm 4.9$ & $0.81(4,9,13)$ & $5.48 \pm 0.2$ & $0.36 \pm 0.01$ \\
\hline Centaurus ............... & 0.011 & $256 \pm 11(5)$ & 3.8 & $0.7(4,9,14,20)$ & 1.1 & 0.060 \\
\hline 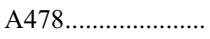 & 0.081 & $\ldots$ & $0.0369 \pm 0.0015$ & $\ldots$ & $0.60 \pm 0.02$ & $0.052 \pm 0.002$ \\
\hline M84 ....................... & 0.0035 & $278 \pm 4(8)$ & $6.00 \pm 0.15$ & $0.63(4,9,17)$ & $0.162 \pm 0.004$ & $0.0089 \pm 0.0002$ \\
\hline 2A $0335+096 \ldots \ldots$ & 0.035 & $\ldots$ & $0.0367 \pm 0.0018$ & $0.9(12)$ & $0.104 \pm 0.005$ & $0.0077 \pm 0.0004$ \\
\hline A262 ..................... & 0.016 & $\ldots$ & $0.0657 \pm 0.0023$ & $0.6(4)$ & $0.039 \pm 0.001$ & $0.00210 \pm 0.00007$ \\
\hline HCG $62 \ldots \ldots \ldots \ldots . . . .$. & 0.014 & $\ldots$ & $0.0049 \pm 0.0005$ & $\ldots$ & $0.0021 \pm 0.0002$ & $0.00018 \pm 0.00002$ \\
\hline
\end{tabular}

${ }^{\text {a }}$ When no velocity dispersion was available, $\langle\sigma\rangle=281 \mathrm{~km} \mathrm{~s}^{-1}$ was adopted. References are in parentheses.

b The spectral index is defined so that $S \sim \nu^{-\alpha}$. When no spectral index was available, $\alpha=1$ was adopted. References are in parentheses.

REFERENCES.-(1) Becker et al. 1991; (2) Bender et al. 1994; (3) Blakeslee \& Tonry 1992; (4) Burbidge \& Crowne 1979; (5) Carollo et al. 1993; (6) Carter et al. 1985; (7) Fisher et al. 1995; (8) Heckman et al. 1985; (9) Kühr et al. 1981; (10) Pedlar et al. 1990; (11) Sarazin et al. 1995b; (12) Sarazin et al. 1995a; (13) Slee 1995; (14) Slee \& Siegman 1988; (15) Slee et al. 2001; (16) Smith et al. 1990; (17) Spinrad et al. 1985; (18) Taylor et al. 1994; (19) Tonry 1985; (20) Wright et al. 1994; (21) Wright et al. 1996.

therefore unlikely to provide a general solution to the heating problem (recent simulations by Dolag et al. 2004 support this conclusion). Additional heat sources, such as cosmic rays (Böhringer \& Morfill 1988; Loewenstein et al. 1991), and supernova explosions (McNamara et al. 2004) may contribute to heating the gas. Nevertheless, these mechanisms are generally incapable of balancing radiation losses.

In this paper, we evaluate whether the mechanical energy generated by active galactic nuclei (AGNs) can balance radiation losses in cluster cores. This possibility, which has a substantial legacy in the literature (e.g., Tabor \& Binney 1993; Binney \& Tabor 1995; Tucker \& David 1997; Ciotti \& Ostriker 2001; Soker et al. 2001), has been rejuvenated by the crisp, new Chandra images of clusters showing the $\mathrm{keV}$ gas being displaced by radio sources harbored by central cluster galaxies. The now ubiquitous signatures of these interactions are X-ray surface brightness depressions projected on the radio lobe emission at $1.4 \mathrm{GHz}$, as is seen in Perseus (Böhringer et al. 1993; Schmidt et al. 2002; Fabian et al. 2000, 2002b, 2003a, 2003b), Cygnus A (Carilli et al. 1994), and Hydra A (McNamara et al. 2000; David et al. 2001; Nulsen et al. 2002). The displacement of the gas creates a low-density, rising bubble in pressure balance with the surrounding medium. X-ray surface brightness depressions that have no obvious association with the bright radio emission at $1.4 \mathrm{GHz}$, the so-called ghost cavities, have also been found, such as those in A2597 (McNamara et al. 2001) and the outer depressions in Perseus (Fabian et al. 2000). These depressions are thought to have been created by interactions that occurred in the in the more distant past, but whose radio emission has faded over time.

This general scenario has been modeled theoretically using a variety of hydrodynamic, magnetohydrodynamic, and analytical techniques, which have successfully reproduced the gross characteristics of the cavities (e.g., Gull \& Northover 1973; Churazov et al. 2001; Brüggen \& Kaiser 2001; Quilis et al. 2001; Brighenti \& Mathews 2002; Brüggen et al. 2002; Reynolds et al. 2002; Brüggen 2003; De Young 2003; Basson \& Alexander 2003; Binney 2004; Kaiser \& Binney 2003; Mathews et al. 2003; Omma et al. 2004; Robinson et al. 2004). The models do not, however, predict with any certainty the amount of mechanical energy provided by radio sources of a given luminosity, nor their frequency of recurrence. Whereas the cavities in several individual objects, such as Perseus (Fabian et al. 2003a) and Hydra A (McNamara et al. 2000), contain enough enthalpy to balance cooling, at least for a short period of time, a systematic survey of cavities in systems with a broad range of properties is required to determine whether this is generally true. In this paper we address this question by setting observational limits on the energetics and ages of cavities in 18 systems taken from the Chandra Data Archive. We adopt $H_{0}=70 \mathrm{~km} \mathrm{~s}^{-1} \mathrm{Mpc}^{-1}, \Omega_{M}=0.3$, and $\Omega_{\Lambda}=0.7$ in all calculations throughout this paper.

\section{THE SAMPLE}

Approximately 80 systems from the Chandra Data Archive were visually inspected for surface brightness depressions. Of these, we selected the 18 systems having well-defined surface brightness depressions associated with their radio sources (see Table 1). Of the 18 systems, 16 were imaged with the ACISS3 detector and two with the ACIS-I3 detector (RBS 797 and MKW 3S), with exposure times ranging from $12 \mathrm{ks}$ (RBS 797 ) to $50 \mathrm{ks}$ (HCG 62). The sample consists of 16 galaxy clusters, one galaxy group (HCG 62), and one giant elliptical galaxy (M84), ranging in redshift from $z=0.0035$ (M84) to $z=0.35$ (RBS 797) and in X-ray luminosity from $\sim 10^{41} \mathrm{ergs}$ $\mathrm{s}^{-1}$ (M84) to $4 \times 10^{45} \mathrm{ergs} \mathrm{s}^{-1}$ (RBS 797). We avoided depressions with questionable association with a radio source, and we excluded clusters in which there is clear evidence of merging, since merging clusters often show complex structure that can be mistaken for a radio-induced cavity. All clusters 
in our sample were previously reported in the literature as containing cavities likely to be associated with radio bubbles.

\section{DATA REDUCTION AND ANALYSIS}

\subsection{Radio Analysis}

The clusters in our sample have a wide range of radio properties, from powerful, double-lobed, FR II type radio sources with luminosities of $\sim 7 \times 10^{44} \mathrm{ergs} \mathrm{s}^{-1}$ (Cygnus A) to weak sources with luminosities of $\sim 2 \times 10^{38} \mathrm{ergs} \mathrm{s}^{-1}$ (HCG 62). Table 1 gives the radio properties of our sample. The radio power at $\nu=1400 \mathrm{MHz}$ was calculated as $P_{\nu}=$ $4 \pi D_{\nu}^{2} S_{\nu}$, where $D_{\nu}=D_{L}(1+z)^{-(1+\alpha) / 2}$ (von Hoerner 1974). The total radio luminosity was calculated by integrating the flux between $\nu_{1}=10 \mathrm{MHz}$ and $\nu_{2}=5000 \mathrm{MHz}$ as

$$
L_{\mathrm{rad}}=4 \pi D_{L}^{2} S_{\nu_{0}} \int_{\nu_{1}}^{\nu_{2}}\left(\nu / \nu_{0}\right)^{-\alpha} d \nu
$$

where we have assumed a power-law spectrum $\left(S_{\nu} \sim \nu^{-\alpha}\right.$, where $\alpha$ is the spectral index). We used for $S_{\nu_{0}}$ the $1400 \mathrm{MHz}$ flux from the NRAO VLA ${ }^{1}$ Sky Survey (NVSS) catalog (Condon et al. 1998), except in the case of the Centaurus Cluster, where no NVSS data were available. In this case, we used the $1410 \mathrm{MHz}$ flux from the Parkes Radio Sources Catalogue (Wright \& Otrupcek 1990). Spectral indices were taken from the catalogs referenced in Table 1. As the derived spectral index can vary depending on the frequencies used, we have adopted a weighted average of the available spectral indices. In cases in which the spectral index was not available, a value of $\alpha=1$ was adopted.

\subsection{X-Ray Analysis}

The X-ray data were obtained through the Chandra Data Archive and were reprocessed with CIAO, version 2.3, using CALDB, version 2.21. The charge transfer inefficiency (CTI) correction was applied during reprocessing of the level 1 event file. Blank-sky background files were used for background subtraction for all clusters. ${ }^{2}$ The background files were normalized to the count rate of the source image in the $10-12 \mathrm{keV}$ band, after the removal of all bright emission. The required adjustment was less than $12 \%$ for all clusters except Centaurus and Perseus, which both required background adjustments of $30 \%$. Spectra with at least 2000 counts were extracted in circular annuli centered on the X-ray centroid of the cluster. Response files were made using the CIAO tools $m \mathrm{krmf}$ and mkwarf. We attempted to correct the resulting ARFs for the quantum efficiency degradation problem using the corrarf tool. ${ }^{3}$ However, upon spectral fitting, we found that $\sim 75 \%$ of our sample was overcorrected by corrarf. Therefore, we present our results without the correction applied (for a discussion of this problem, see Voigt \& Fabian 2004). In general, the largest effect of using corrarf was on the cooling rates, which increased on average by a factor of 2 after the correction was applied.

To find radial temperatures and densities, we deprojected the spectra extracted above. The deprojection was performed

\footnotetext{
${ }^{1}$ The VLA (Very Large Array) is a facility of the National Radio Astronomy Observatory (NRAO). The NRAO is a facility of the National Science Foundation operated under cooperative agreement by Associated Universities, Inc.

${ }^{2}$ See http://asc.harvard.edu/contrib/maxim/acisbg.

3 See http://asc.harvard.edu/cal/Acis/Cal_prods/qeDeg
}

assuming spherical symmetry and using the PROJCT model in XSPEC, version 11.2.0, with a single-temperature plasma model (MEKAL) and foreground absorption (WABS), fitted between energies of 0.5 and $7.0 \mathrm{keV}$. The foreground column density, $N_{\mathrm{H}}$, was tied between annuli and allowed to vary. The MEKAL abundance was also free to vary. The redshift was fixed to the value given in Table 1 . The density was then calculated from the normalization of the MEKAL component, assuming $n_{\mathrm{e}}=1.2 n_{\mathrm{H}}$ (for a fully ionized gas with hydrogen and helium mass fractions of $X=0.7$ and $Y=0.28$ ). The pressure in each annulus was calculated as $P=n k T$, where we have assumed an ideal gas and $n \approx 2 n_{e}$.

The luminosity of the cooling gas inside the cooling radius is needed to investigate whether or not AGN heating can balance cooling. Within the cooling radius, radiative energy losses must be replaced to prevent the deposition of large quantities of cool gas. We define the cooling radius as the radius within which the gas has a cooling time less than $7.7 \times 10^{9} \mathrm{yr}$, the look-back time to $z=1$ for our adopted cosmology. This redshift is roughly the distance to which clusters have been found with properties similar to present-day clusters. The corresponding look-back time should then approximate the time a cooling flow has had to establish itself. The cooling time was calculated using the cooling curves of Böhringer \& Hensler (1989). Table 2 gives the values of $r_{\text {cool }}$ for each cluster. Within this radius, we performed deprojections by fitting both a cooling model and a single-temperature model to the spectra.

In order to obtain a spectroscopic estimate of the cooling luminosity, we performed the deprojection using a cooling flow model (PROJCT $\times$ WABS $\times[$ MEKAL + MKCFLOW]), fitted between 0.5 and $7.0 \mathrm{keV}$. To force all cooling to be within the cooling radius, the MKCFLOW model was used only inside the cooling radius and was set to zero outside. The MKCFLOW low temperature was fixed to $0.1 \mathrm{keV}$, resulting in an estimate of the luminosity of gas cooling to low temperatures. Within each annulus, the MEKAL and MKCFLOW abundances were tied together, and the MKCFLOW high temperature was tied to the temperature of the MEKAL component. Lastly, the column density $\left(N_{\mathrm{H}}\right)$ was tied between annuli and allowed to vary. The spectroscopic estimate of the bolometric cooling luminosity inside the cooling radius, $L_{\text {spec }}$, was then calculated from the unabsorbed fluxes obtained from the MKCFLOW model, integrated between energies of $\sim 0.1$ and $100 \mathrm{keV}$.

To find the total luminosity inside the cooling radius, we performed the deprojection using a single-temperature model $(\mathrm{PROJCT} \times \mathrm{WABS} \times \mathrm{MEKAL}$ ) fitted between 0.5 and $7.0 \mathrm{keV}$ to the same spectra used with the cooling flow model, again with $N_{\mathrm{H}}$ tied between regions and allowed to vary. The unabsorbed fluxes from the MEKAL components for the annuli within the cooling radius, extrapolated between $\sim 0.1$ and $100 \mathrm{keV}$, were used to find the bolometric luminosity of the X-ray-emitting gas, $L_{\mathrm{X}}$. Table 2 gives $L_{\mathrm{X}}$ and $L_{\text {spec }}$ (with $1 \sigma$ errors estimated by XSPEC) for each object in our sample. Typically, $L_{\mathrm{spec}}$ is approximately $10 \%$ of $L_{\mathrm{X}}$ for our sample. Our values for $L_{\text {spec }}$ and $L_{\mathrm{X}}$ are in reasonable agreement with published values for most of our sample.

\section{X-RAY SURFACE BRIGHTNESS DEPRESSIONS}

In total, 36 surface brightness depressions or cavities were identified in the 18 systems. Table 3 lists the cavity properties. The cavities are classified as either radio-filled or radio-faint ghosts, depending on the presence of $1400 \mathrm{MHz}$ or higher 
TABLE 2

Results of X-Ray Spectral Modeling

\begin{tabular}{|c|c|c|c|c|c|c|}
\hline \multirow[b]{2}{*}{ System } & \multirow[b]{2}{*}{$\begin{array}{l}r_{\mathrm{cool}} \\
(\mathrm{kpc})\end{array}$} & \multicolumn{2}{|c|}{$\mathrm{PROJCT} \times \mathrm{WABS} \times \mathrm{MEKAL}$} & \multicolumn{3}{|c|}{$\mathrm{PROJCT} \times \mathrm{WABS} \times(\mathrm{MEKAL}+\mathrm{MKCFLOW})$} \\
\hline & & $\chi^{2} / \mathrm{dof}$ & $\begin{array}{c}L_{\mathrm{X}}\left(<r_{\text {cool }}\right) \\
\left(10^{42} \mathrm{ergs} \mathrm{s}^{-1}\right)\end{array}$ & $\chi^{2 / \mathrm{dof}}$ & $\begin{array}{c}\dot{M} \\
\left(M_{\odot} \mathrm{yr}^{-1}\right)\end{array}$ & $\begin{array}{c}L_{\text {spec }}\left(<r_{\text {cool }}\right) \\
\left(10^{42} \operatorname{ergs~s}^{-1}\right)\end{array}$ \\
\hline RBS $797 \ldots \ldots \ldots \ldots \ldots . . . . .$. & 191 & $154 / 183$ & $4500_{-700}^{+800}$ & $151 / 180$ & $880_{-670}^{+1800}$ & $1200_{-1100}^{+4600}$ \\
\hline $\mathrm{A} 478 \ldots \ldots \ldots \ldots \ldots \ldots$ & 150 & $3704 / 2796$ & $1220 \pm 60$ & $3671 / 2792$ & $150_{-68}^{+60}$ & $180_{-95}^{+60}$ \\
\hline Perseus ................... & 102 & $9348 / 5717$ & $670_{-30}^{+40}$ & $9165 / 5703$ & $54_{-18}^{+48}$ & $59_{-17}^{+31}$ \\
\hline A1795....................... & 137 & $2476 / 1721$ & $490 \pm 30$ & $2487 / 1718$ & $18_{-10}^{+12}$ & $11_{-5}^{+9}$ \\
\hline 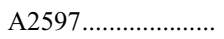 & 129 & $1341 / 1149$ & $430_{-30}^{+40}$ & $1329 / 1145$ & $59 \pm 40$ & $28 \pm 19$ \\
\hline Cygnus A ................ & 78 & $3062 / 2234$ & $410 \pm 30$ & $3062 / 2230$ & $<8$ & $<6$ \\
\hline $2 \mathrm{~A} 0335+096 \ldots \ldots$. & 122 & $2439 / 1903$ & $290 \pm 20$ & $2357 / 1897$ & $120 \pm 30$ & $44_{-11}^{+12}$ \\
\hline Hydra A ................. & 100 & $1658 / 1486$ & $250 \pm 15$ & $1652 / 1482$ & $14_{-7}^{+9}$ & $8.1_{-3.7}^{+4.8}$ \\
\hline 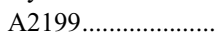 & 113 & $2422 / 1971$ & $150 \pm 10$ & $2421 / 1966$ & $2.0_{-1.9}^{+7.0}$ & $1.2_{-1.1}^{+4.5}$ \\
\hline A133 & 92 & $1624 / 1160$ & $95 \pm 5$ & $1598 / 1158$ & $25 \pm 6$ & $11 \pm 3$ \\
\hline MKW 3S.................. & 88 & $2242 / 2037$ & $92 \pm 7$ & $2242 / 2033$ & $<2$ & $<1$ \\
\hline A2052 ...................... & 101 & $2934 / 2035$ & $84_{-5}^{+6}$ & $2760 / 2029$ & $81_{-11}^{+15}$ & $22_{-5}^{+9}$ \\
\hline A4059........................ & 99 & $1396 / 1109$ & $71_{-7}^{+8}$ & $1296 / 988$ & $6.7_{-4.1}^{+8.5}$ & $4.7_{-3.3}^{+3.9}$ \\
\hline Centaurus .................. & 62 & $5100 / 2177$ & $30 \pm 2$ & $4932 / 2171$ & $10.2_{-1.2}^{+1.5}$ & $2.8_{-0.4}^{+0.6}$ \\
\hline A $262 \ldots \ldots \ldots \ldots \ldots \ldots \ldots$ & 66 & $1978 / 1169$ & $13.8 \pm 1.0$ & $1923 / 1165$ & $1.5_{-0.4}^{+0 . .^{2}}$ & $1.5_{-0.4}^{+0.4}$ \\
\hline M87 ............ & 35 & $15540 / 5714$ & $9.8_{-0.7}^{+0.8}$ & $15622 / 5688$ & $1.8_{-0.6}^{+1.4}$ & $0.62_{-0.18}^{+0.44}$ \\
\hline HCG $62 \ldots \ldots \ldots \ldots \ldots$ & 33 & $1008 / 626$ & $2.0 \pm 0.2$ & $961 / 624$ & $1.1_{-0.2}^{+0.3}$ & $0.20_{-0.03}^{+0.04}$ \\
\hline M84 ...................... & 14 & $682 / 450$ & $0.09 \pm 0.01$ & $701 / 448$ & $0.06 \pm 0.05$ & $0.017_{-0.004}^{+0.016}$ \\
\hline
\end{tabular}

frequency radio emission inside the cavity. The cavities are classified as radio-filled if there is a direct anticorrelation between the radio emission at $1400 \mathrm{MHz}$ or higher and the $\mathrm{X}$-ray emission, such that the radio emission fills preferentially the X-ray surface brightness depressions. The cavities classified as ghost cavities, while possibly possessing significant radio emission at frequencies at or below $1400 \mathrm{MHz}$, do not show the anticorrelation between the high-frequency radio emission and the $\mathrm{X}$-ray emission. Our classification scheme relies heavily on the availability of high-resolution radio images at several frequencies. However, the radio data available are inhomogeneous, and classifying the objects lacking high-resolution radio images was challenging. The poor radio images available for A262, RBS 797, and HCG 62 , in particular, led us to classify them as ghosts (see E. L. Blanton et al. 2004, in preparation).

For each cavity, a size and position were measured, assuming that the cavity extends to the inner edge of any bright surrounding emission. The projected shapes of the cavities were measured by eye as circles or ellipses from the exposurecorrected, unsmoothed images. This is a qualitative measurement, the accuracy of which depends on the signal-to-noise ratio of the image and on the contrast of the cavity with its surroundings. To distinguish between the poorly defined and well-defined cavities, we have assigned a figure of merit (FOM) to each cavity, ranging from 1 for the best-defined cavities - those with surrounding bright rims - to 3 for the worst-defined ones without bright rims.

In the analysis that follows, we assumed that the cavities are bubbles devoid of gas at the local ambient temperature (McNamara et al. 2000; Blanton et al. 2003). Their volumes were calculated assuming spherical or prolate ellipsoidal shapes, with semimajor axis $a$ and semiminor axis $b$. The errors in the volumes due to projection were estimated by allowing each bubble to have an intrinsic $a / b$ as large as that of the most eccentric cavity observed in the sample, $(a / b)_{\max }$. The upper and lower limits are calculated assuming either oblate or prolate symmetry. In this sense, spherical bubbles have the greatest range of possible volumes, while projected ellipses with an $a / b=(a / b)_{\max }^{2 / 3}$ have the smallest range. The pressure and temperature of the gas surrounding the cavity were taken to be the azimuthally averaged values at the projected radius of its center. The work done on the surrounding medium by the cavity is then simply $W_{\text {bub }}=p V$, if it expands slowly compared to the sound speed.

\subsection{Cavity Ages}

The age of each cavity was calculated in three ways. First, we calculated the time required for the cavity to rise the projected distance from the radio core to its present location at the speed of sound, $v_{c_{s}}=\left(\gamma k T / \mu m_{\mathrm{H}}\right)^{1 / 2}$, where we have taken $\gamma=5 / 3$ and $\mu=0.62$. The cavity age is then

$$
t_{c_{s}}=R / v_{c_{s}}=R \sqrt{\mu m_{\mathrm{H}} / \gamma k T}
$$

where $R$ is the projected distance from the center of the bubble to the radio core. This scenario is favored in the computational modeling of Omma et al. (2004), in which the bubble is produced by a high-momentum jet from the AGN instead of rising buoyantly. Second, the age was calculated as the time required for the cavity to rise buoyantly (bubble-like) at its terminal velocity $v_{t} \sim(2 g V / S C)^{1 / 2}$, where $V$ is the volume of the bubble, $S$ is the cross section of the bubble, and $C=0.75$ is the drag coefficient (Churazov et al. 2001). The gravitational acceleration was calculated using the stellar velocity dispersion of the central galaxy, under the approximation that the galaxy is an isothermal sphere, as $g \approx 2 \sigma^{2} / R$ (Binney \& Tremaine 1987). Published values of the velocity dispersion were used when available (see Table 1); otherwise, the average value $\left(\langle\sigma\rangle=289 \mathrm{~km} \mathrm{~s}^{-1}\right)$ was adopted. The cavity age is then given by

$$
t_{\text {buoy }}=R / v_{t} \sim R \sqrt{S C / 2 g V} .
$$


TABLE 3

Cavity Properties

\begin{tabular}{|c|c|c|c|c|c|c|c|c|c|}
\hline System & Cavity Type (FOM) ${ }^{\mathrm{a}}$ & $\begin{array}{c}a^{\mathrm{b}} \\
(\mathrm{kpc})\end{array}$ & $\begin{array}{c}b^{\mathrm{c}} \\
(\mathrm{kpc})\end{array}$ & $\begin{array}{c}R^{\mathrm{d}} \\
(\mathrm{kpc})\end{array}$ & $\begin{array}{c}p V^{\mathrm{e}} \\
\left(10^{57} \mathrm{ergs}\right)\end{array}$ & $\begin{array}{c}t_{c_{s}} \\
\left(10^{7} \mathrm{yr}\right)\end{array}$ & $\begin{array}{c}t_{\text {buoy }} \\
\left(10^{7} \mathrm{yr}\right)\end{array}$ & $\begin{array}{c}t_{r} \\
\left(10^{7} \mathrm{yr}\right)\end{array}$ & References \\
\hline \multirow[t]{2}{*}{ RBS $797 \ldots \ldots \ldots \ldots . . . .}$. & $\mathrm{G}(2)$ & 9.7 & 9.7 & 19.5 & $190_{-120}^{+340}$ & 1.4 & 3.6 & 6.8 & \multirow[t]{2}{*}{18} \\
\hline & $\mathrm{G}(2)$ & 13.4 & 8.5 & 23.8 & $190_{-40}^{+150}$ & 1.7 & 5.2 & 7.8 & \\
\hline \multirow[t]{2}{*}{ A478.................... } & $\mathrm{F}(2)$ & 5.5 & 3.4 & 9.0 & $5.8_{-1.1}^{+4.4}$ & 1.1 & 1.9 & 3.1 & \multirow[t]{2}{*}{20} \\
\hline & $\mathrm{F}(2)$ & 5.6 & 3.4 & 9.0 & $6.0_{-1.1}^{+4.1}$ & 1.1 & 1.9 & 3.1 & \\
\hline A1795 ................... & G (3) & 18.5 & 7.2 & 18.5 & $39_{-4}^{+53}$ & 1.8 & 3.7 & 6.8 & 5 \\
\hline \multirow[t]{4}{*}{ Perseus ................ } & $\mathrm{F}(1)$ & 4.7 & 4.7 & 7.0 & $12_{-8}^{+21}$ & 0.6 & 1.3 & 3.2 & \multirow[t]{4}{*}{6,7} \\
\hline & $\mathrm{F}(1)$ & 7.3 & 6.3 & 11.2 & $24_{-13}^{+34}$ & 1.0 & 2.1 & 4.8 & \\
\hline & $\mathrm{G}(1)$ & 12.9 & 6.3 & 29.7 & $24_{-1}^{+13}$ & 3.0 & 9.4 & 9.1 & \\
\hline & $\mathrm{G}(2)$ & 13.6 & 4.8 & 36.7 & $14_{-1}^{+125}$ & 3.6 & 15.1 & 9.7 & \\
\hline \multirow{2}{*}{ Cygnus A ............. } & $\mathrm{F}(1)$ & 29.0 & 17.2 & 43.0 & $470_{-30}^{+300}$ & 3.2 & 8.9 & 15.3 & \multirow[t]{2}{*}{19} \\
\hline & $\mathrm{F}(1)$ & 33.8 & 23.1 & 44.7 & $930_{-230}^{+860}$ & 3.3 & 8.1 & 17.4 & \\
\hline \multirow{2}{*}{ A2597................... } & $\mathrm{G}(2)$ & 10.2 & 7.1 & 22.6 & $21_{-5}^{+20}$ & 2.6 & 6.6 & 8.6 & \multirow[t]{2}{*}{15} \\
\hline & $\mathrm{G}(2)$ & 7.1 & 7.1 & 23.1 & $14_{-9}^{+26}$ & 2.6 & 6.8 & 7.9 & \\
\hline \multirow[t]{2}{*}{ 2A $0335+096 \ldots .}$. & $\mathrm{G}(2)$ & 9.3 & 6.5 & 23.1 & $9.7_{-2.8}^{+9.6}$ & 3.2 & 5.7 & 6.6 & \multirow[t]{2}{*}{13} \\
\hline & G (3) & 4.8 & 2.6 & 27.5 & $0.79_{-0.02}^{+0.40}$ & 3.9 & 11.7 & 4.8 & \\
\hline \multirow[t]{2}{*}{ Hydra A .................. } & $\mathrm{F}(2)$ & 17.7 & 11.8 & 28.5 & $83_{-17}^{+73^{02}}$ & 3.0 & 5.1 & 8.7 & \multirow[t]{2}{*}{$4,14,16$} \\
\hline & $F(2)$ & 19.9 & 11.7 & 33.8 & $87_{-7}^{+57}$ & 3.2 & 5.6 & 9.3 & \\
\hline \multirow[t]{2}{*}{ A2199................ } & $\mathrm{F}(2)$ & 6.5 & 6.5 & 18.9 & $6.6_{-4.3}^{+11.9}$ & 2.1 & 4.0 & 5.2 & \multirow[t]{2}{*}{11} \\
\hline & $\mathrm{F}(2)$ & 6.2 & 3.5 & 21.2 & $1.8_{-0.1}^{+1.1}$ & 2.2 & 6.5 & 4.7 & \\
\hline MKW 3S............. & G (3) & 53.9 & 22.6 & 58.7 & $300_{-14}^{+310}$ & 5.8 & 12.4 & 22.3 & 12 \\
\hline \multirow[t]{2}{*}{ 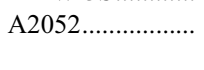 } & $\mathrm{F}(1)$ & 6.5 & 6.0 & 6.7 & $3.2_{-1.8}^{+5.0}$ & 1.1 & 1.0 & 3.5 & \multirow[t]{2}{*}{1,2} \\
\hline & $\mathrm{F}(1)$ & 10.7 & 7.8 & 11.2 & $8.4_{-2.8}^{+8.8}$ & 1.8 & 2.0 & 5.5 & \\
\hline \multirow[t]{2}{*}{ A4059.................. } & $\mathrm{G}(2)$ & 9.2 & 9.2 & 19.3 & $8.9_{-6.0}^{+16.8}$ & 2.8 & 3.5 & 6.2 & \multirow[t]{2}{*}{10} \\
\hline & $\mathrm{G}(2)$ & 20.4 & 10.1 & 22.7 & $24_{-3}^{-0.0}$ & 2.6 & 4.2 & 8.4 & \\
\hline \multirow[t]{2}{*}{ A133.................... } & $\mathrm{F}(2)$ & 9.8 & 5.2 & 28.1 & $3.4_{-0}^{+3.7}$ & 3.5 & 8.6 & 7.0 & \multirow[t]{2}{*}{9} \\
\hline & $\mathrm{F}(2)$ & 9.8 & 5.7 & 32.7 & $4.0_{-0.1}^{+2.1}$ & 3.9 & 10.2 & 7.7 & \\
\hline \multirow[t]{2}{*}{ Centaurus ............ } & $\mathrm{F}(3)$ & 3.3 & 1.6 & 3.5 & $0.196_{-0.003}^{+0.10}$ & 0.7 & 0.7 & 1.5 & \multirow[t]{2}{*}{17} \\
\hline & $\mathrm{F}(2)$ & 3.3 & 2.4 & 6.0 & $0.40_{-0.13}^{+0.42}$ & 1.0 & 1.3 & 2.2 & \\
\hline \multirow[t]{2}{*}{ A $262 .}$. & G (2) & 2.6 & 2.6 & 6.2 & $0.17_{-0.11}^{+0.32}$ & 1.1 & 1.3 & 2.0 & \multirow[t]{2}{*}{3} \\
\hline & G (3) & 3.3 & 2.6 & 6.7 & $0.21_{-0.10}^{+0.27}$ & 1.2 & 1.4 & 2.2 & \\
\hline M87 ....................... & $\mathrm{F}(2)$ & 1.6 & 0.8 & 2.2 & $0.078_{-0.003}^{+0.043}$ & 0.4 & 0.4 & 0.6 & 22 \\
\hline & $F(2)$ & 2.3 & 1.4 & 2.8 & $0.25_{-0.02}^{+0.18}$ & 0.4 & 0.4 & 0.9 & \\
\hline HCG $62 \ldots \ldots \ldots \ldots . . . .$. & G (2) & 5.0 & 4.3 & 8.4 & $0.29_{-0.15}^{+0.41}$ & 1.8 & 1.5 & 3.1 & 21 \\
\hline & $G(2)$ & 4.0 & 4.0 & 8.6 & $0.21_{-0.13}^{+0.37}$ & 1.9 & 1.6 & 2.9 & \\
\hline M84 ....................... & $\mathrm{F}(2)$ & 1.6 & 1.6 & 2.3 & $0.019_{-0.013}^{+0.035}$ & 0.5 & 0.4 & 1.0 & 8 \\
\hline & $F(2)$ & 2.1 & 1.2 & 2.5 & $0.013_{-0.001}^{+0.007}$ & 0.6 & 0.5 & 1.0 & \\
\hline
\end{tabular}

a Radio-filled cavities are denoted by "F," and radio-faint ghosts are denoted by "G." The FOM gives a relative measure of the cavity's contrast to its surroundings: (1) high contrast: bright rim surrounds cavity; (2) medium contrast: bright rim partially surrounds cavity; and (3) low contrast: no rim, or faint rim surrounds cavity.

b Projected semimajor axis of the cavity.

c Projected semiminor axis of the cavity.

${ }^{\mathrm{d}}$ Projected distance from the cavity center to the radio core.

e The errors in $p V$ include an estimate of the projection effects; see the text for details.

References.- (1) Blanton et al. 2001; (2) Blanton et al. 2003; (3) E. L. Blanton et al. 2004, in preparation; (4) David et al. 2001; (5) Ettori et al. 2002; (6) Fabian et al. 2000; (7) Fabian et al. 2002a; (8) Finoguenov \& Jones 2001 (9) Fujita et al. 2002; (10) Heinz et al. 2002; (11) Johnstone et al. 2002; (12) Mazzotta et al. 2002; (13) Mazzotta et al. 2003; (14) McNamara et al. 2000; (15) McNamara et al. 2001; (16) Nulsen et al. 2002; (17) Sanders \& Fabian 2002; (18) Schindler et al. 2001; (19) Smith et al. 2002; (20) Sun et al. 2003; (21) Vrtilek et al. 2002; (22) Young et al. 2002.

Finally, the age was calculated as the time required to refill the displaced volume as the bubble rises upward (McNamara et al. 2000; Nulsen et al. 2002):

$$
t_{r} \sim 2 R \sqrt{r / G M(R)}=2 \sqrt{r / g},
$$

where $r$ is the radius of the cavity [for ellipsoidal cavities, $\left.r=(a b)^{1 / 2}\right]$.

In general, the ages calculated using the speed of sound are the shortest, those based on the refilling timescale are the greatest, and those calculated using the terminal velocity lie in between. The instantaneous mechanical luminosity per cavity or cavity pair is then $L_{\text {mech }}=W_{\text {bub }} / t$, where $t$ is the age of the bubble.
This approximation to the mechanical luminosity of the rising bubbles is highly uncertain. In calculating $L_{\text {mech }}$, a measurement of the average timescale for the radio source to replenish itself is required. This timescale is unknown for each source, but perhaps this can best be measured in objects with clearly defined ghost cavities and a detached central source, where the duty cycle is clearly evident. This situation has been noted in two objects, Perseus (Fabian et al. 2000) and A2597 (McNamara et al. 2001), whose ghost cavities range in age between $\sim 5 \times 10^{7}$ and $\sim 8 \times 10^{7} \mathrm{yr}$, with a likely age of $\sim 10^{8} \mathrm{yr}$ when projection is taken into account. Furthermore, of the 80 or so clusters we searched for cavities, 16 were found to have them. If one assumes, for the moment, that all central cluster galaxies produce bubbles at a similar rate, then 

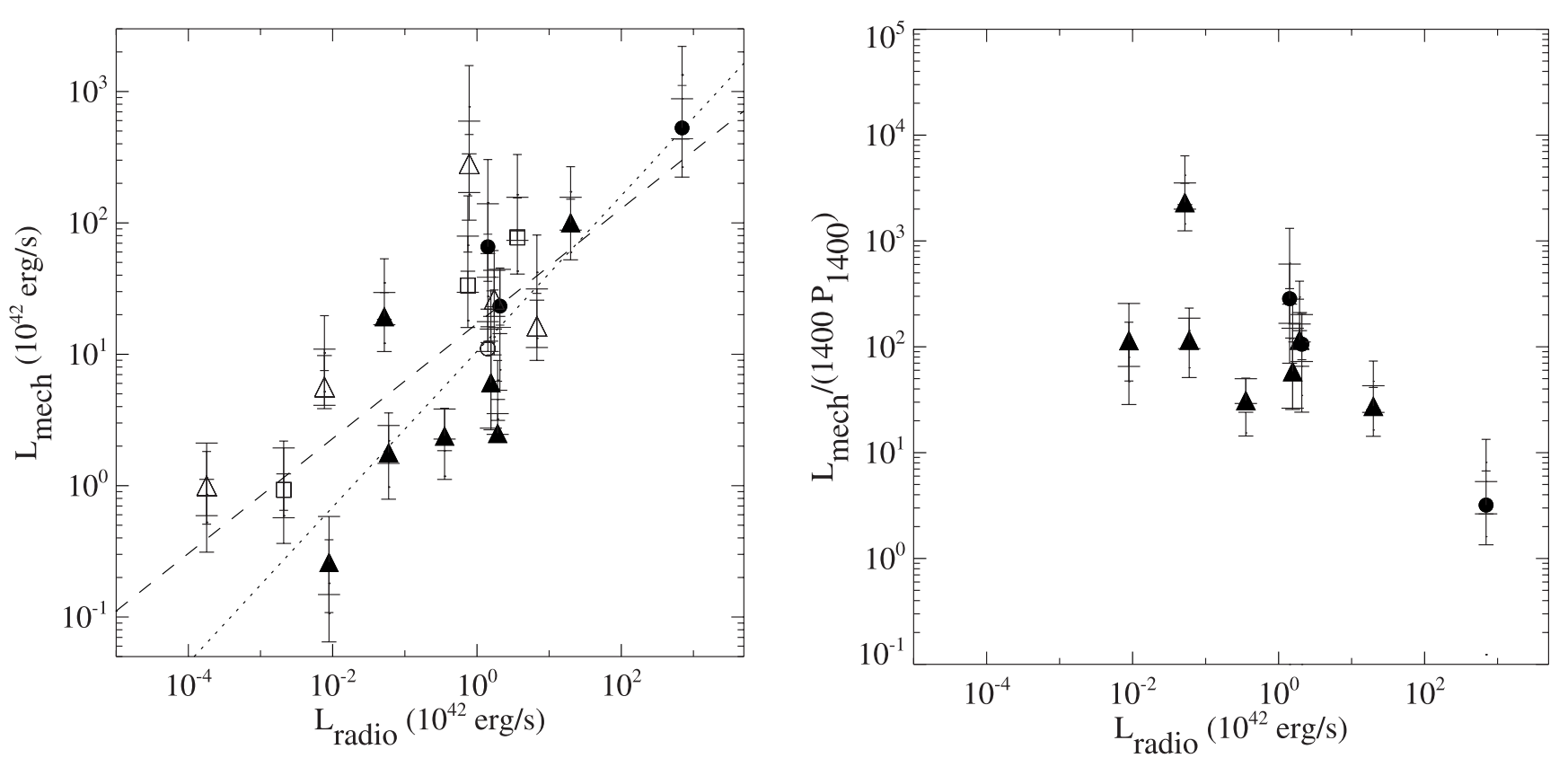

FIG. 1. Left: Mechanical vs. radio luminosity. The symbols and wide error bars denote the values of the mechanical luminosity calculated using the buoyancy timescale. The short and medium-width error bars denote upper and lower limits of the mechanical luminosity calculated using the sound speed and refill timescales, respectively. The symbols indicate FOMs of 1 (circles), 2 (triangles), and 3 (squares). Filled symbols denote radio-filled cavities, and open symbols denote ghost cavities. Each point represents the sum of mechanical luminosities of each bubble type. The best-fit lines are shown for the entire sample (dashed line) and for the radio-filled cavities only (dotted line). Right: Ratio of mechanical luminosity to $\nu P_{\nu}$ at $1400 \mathrm{MHz}$ vs. radio luminosity for the radio-filled cavities only.

we are seeing clusters in an on state only $\sim 20 \%$ of the time. Therefore, the average time elapsed between the production of bubble pairs could be as large as $5 \times 10^{8} \mathrm{yr}$, at least in some clusters. Our search did not discriminate between cooling and noncooling clusters, and it is likely, although it has not been proved, that bubbles are produced preferentially in cooling flows. If so, our estimate of $\sim 10^{8} \mathrm{yr}$ between outbursts is probably closer to the truth for most cooling flow clusters. Then the time averaged mechanical luminosity discussed below has been overestimated in most objects by factors of 2-3. On the other hand, the $333 \mathrm{MHz}$ radio map of Hydra A (Lane et al. 2004) shows $2^{\prime}$ plumes extending several times farther from the AGN than the X-ray cavities (and the $4 \mathrm{GHz}$ radio image). It also shows an outer radio lobe $4^{\prime}$ north of the AGN that coincides with a feature in the X-ray image that hints of a distant cavity. Interpreted as above, the outer feature would give a very long interval $\left(\sim 10^{9} \mathrm{yr}\right)$ between bubbles, but the plume indicates that such an outer bubble has been followed (perhaps some time later) by an extended period of continuous radio activity. In that case, the cavities may just be the latest in a series, but we are failing to detect most of the remnants. This would make our estimate of $L_{\text {mech }}$ closer to the truth.

\section{DISCUSSION}

\subsection{Trends with Radio Luminosity}

In Figure 1, we present two plots showing the mechanical luminosity versus the total radio luminosity (left) and the total radio luminosity versus the ratio of the mechanical luminosity to the monochromatic, $1.4 \mathrm{GHz}$ radio luminosity (right). In each plot we distinguish between radio-filled and ghost cavities, shown with filled and open symbols, respectively. The "error bars" for each point reflect the range of instantaneous mechanical luminosity implied by the range in possible ages. The data are taken or are derived from Tables 1 and 3 .
The left-hand panel of Figure 1 shows a trend between the radio luminosity and mechanical luminosity, with the sense that more luminous radio sources tend toward larger mechanical luminosities. This trend seems to be shared by both the radio-filled cavities and the ghost cavities, in spite of the use of the current central radio power for both the filled cavities and the ghosts, to which the current central source may be unrelated. No segregation by FOM is seen. The relation between the two luminosities appears to be roughly a power law. To quantify this relation, we used a linear leastsquares fit to the logarithms of the data, with errors in mechanical luminosity given by the extreme values for each system. We show in Figure 1 the best-fit lines for the entire sample (dashed line), given by

$$
L_{\text {mech }}=10^{25 \pm 3}\left(L_{\text {radio }}\right)^{0.44 \pm 0.06}
$$

and for the radio-filled cavities only (dotted line), given by

$$
L_{\text {mech }}=10^{18 \pm 4}\left(L_{\text {radio }}\right)^{0.6 \pm 0.1}
$$

In both cases, the mechanical luminosity scales as the radio luminosity to approximately the one-half power over six decades of radio power, albeit with large scatter.

The relative contribution of cosmic scatter and observational uncertainty is hard to judge without precision radio data at a variety of wavelengths and without a better understanding of the bubble production timescale. Nevertheless, the existence of this trend demonstrates quantitatively that the radio sources are indeed creating the cavities. The radio sources are not simply filling preexisting voids in the intracluster medium (ICM) created by other processes. Furthermore, the synchrotron luminosity and mechanical luminosity do not scale in direct proportion to each other. This relationship implies that 
the synchrotron luminosity cannot be used to infer the mechanical power of a radio jet in a simple fashion.

An important and poorly understood aspect of radio source physics is the degree of coupling between the mechanical (kinetic) luminosity of radio sources and their synchrotron luminosity. This coupling is theoretically tied to the magnetic field strength and age of the source (see De Young 1993; Bicknell et al. 1997), neither of which can be measured reliably from radio data alone. Radio sources are inefficient radiators. The ratio of mechanical power to radio power is typically assumed to range between 10 and 100 , almost entirely on the basis of theoretical considerations (De Young 1993; Bicknell et al. 1997). On the other hand, measurements of the X-ray cavity sizes and surrounding gas pressures provide unique estimates of their ages and mechanical luminosities, independently of the radio properties themselves. We evaluate the ratio of mechanical energy to radio power by plotting the ratio of mechanical power in the bubbles to monochromatic, $1.4 \mathrm{GHz}$ synchrotron luminosity, assuming $1 \mathrm{pV}$ of energy per radio lobe, against the radio luminosity in the right-hand panel of Figure 1. This ratio ranges from a few to a few hundred for the powerful sources, which is broadly consistent with theoretical estimates (see De Young 1993; Bicknell et al. 1997). On the other hand, A478 has a ratio exceeding a few thousand. To the extent that X-ray cavities provide a good measure of the mechanical energy of radio sources, the large variation in this ratio indicates that radio luminosity is not necessarily a reliable probe of the available mechanical energy.

There are several factors that can introduce scatter into our estimate of the ratio of radio to kinetic power. The most important is probably intrinsic differences between the radio sources themselves, a consequence of dramatic changes in radio luminosity with time. Certainly, if radio outbursts are to compensate for radiative losses in cooling flows, then the absence of radio emission from some systems requires large variations of radio luminosity with time. On the other hand, the $p V$ energy of the bubbles alone would tend to underestimate the mechanical luminosity of radio sources by factors of several if energy dissipating shocks are generated, or if the bubbles expand nonadiabatically (they leak), or if the internal energy of the bubbles is boosted with a relativistic plasma.

\subsection{Heating by Radio-induced Cavities}

Churazov et al. (2002) noted the conversion of enthalpy of the rising bubble into other forms in the cluster atmosphere. Here it is shown that, for an adiabatic bubble, this energy is dissipated in its wake. If the mass in the bubble is negligible compared to the mass of the gas it displaces, then a bubble rises, because the gas falls in around it to fill the space it occupied. This process is driven by the potential energy released as the surrounding gas moves inward. The energy is first converted to gas kinetic energy, then dissipated in the wake of the rising bubble. In the notation of $\S 4.1$, the potential energy released when the bubble rises a small distance, $\delta R$, is

$$
\delta W=\rho V g \delta R=-V \frac{d p}{d R} \delta R
$$

where $\rho$ is the gas density, and we have used the equation of hydrostatic equilibrium to replace $\rho g=-d p / d R$, where $p$ is the gas pressure. This gives a differential equation for the energy dissipated in the bubble wake,

$$
\frac{d W}{d R}=-V \frac{d p}{d R}
$$

If the bubble is adiabatic, with ratio of specific heats $\gamma$, then $p V^{\gamma}=$ constant, and this equation can be integrated to give the energy dissipated as the bubble rises over a large distance, from $R_{0}$ to $R_{1}$,

$$
\Delta W=\frac{\gamma}{\gamma-1}\left(p_{0} V_{0}-p_{1} V_{1}\right)=H_{0}-H_{1}
$$

Here subscripts 0 and 1 label quantities at the corresponding radii, and the enthalpy of the bubble is $H=\gamma p V /(\gamma-1)$. Note that the bubble is assumed to be small compared to $R$ (otherwise, there can be a significant change in the density of the gas as it falls in around the bubble). This would rarely be significant in a cluster, but when it is, then some of the potential energy goes into readjustment of the atmosphere as the bubble moves.

For a relativistic gas, $\gamma=4 / 3$, so that the enthalpy is $4 p V$. The region where this is dissipated by an adiabatic bubble is determined by the pressure distribution of the atmosphere. For clusters such as Hydra A and Perseus, roughly half of this energy would be dissipated inside the cooling radius. It is likely that the bubbles are not entirely adiabatic. On the basis of our numbers, radio losses are generally negligible, but pieces may be broken away from bubbles, and the relativistic particles may leak. Such effects will generally lead to a greater proportion of the bubble energy being deposited within the cooling radius.

It is important to note that our estimate of the mechanical luminosity relies critically on the assumption that the bubbles are close to local pressure equilibrium. This is at least approximately true for the Hydra A Cluster (Nulsen et al. 2002). However, according to the standard view of radio sources, bubbles may have been significantly overpressured while being formed (e.g., Heinz et al. 1998). In that case, the expanding bubble drives a shock, and the energy deposited by the expansion can be substantially larger than $p V$.

There may be additional heat input from the AGNs associated with radio outbursts. This could take the form of spherical shocks (driven by poorly collimated outflows), direct injection of relativistic particles, inverse Compton heating (Ciotti \& Ostriker 2001), or other processes. Very substantial additional heat inputs would drive convection, leading to an isentropic core and mixing out abundance gradients (Brüggen 2002), but this is not a very strong constraint. If such energy injection is significantly more than the bubble energy input, then it is inappropriate to associate it directly with the bubbles, but the mean heating power may be correlated with bubble mechanical power.

Finally, it should be noted that even for adiabatic bubbles, the free energy of a bubble decreases with time, and bubbles may even break up quickly, so that they disappear as X-ray cavities. This means that the instantaneous estimate of bubble mechanical power that we have used varies with time and may vary dramatically. A much better controlled sample is needed to investigate such issues.

\subsection{Can Cavity Production Quench Cooling Flows?}

We now turn to the question of whether radio sources deposit enough energy into the ICM to quench cooling. We use $L_{\mathrm{X}}$, the 


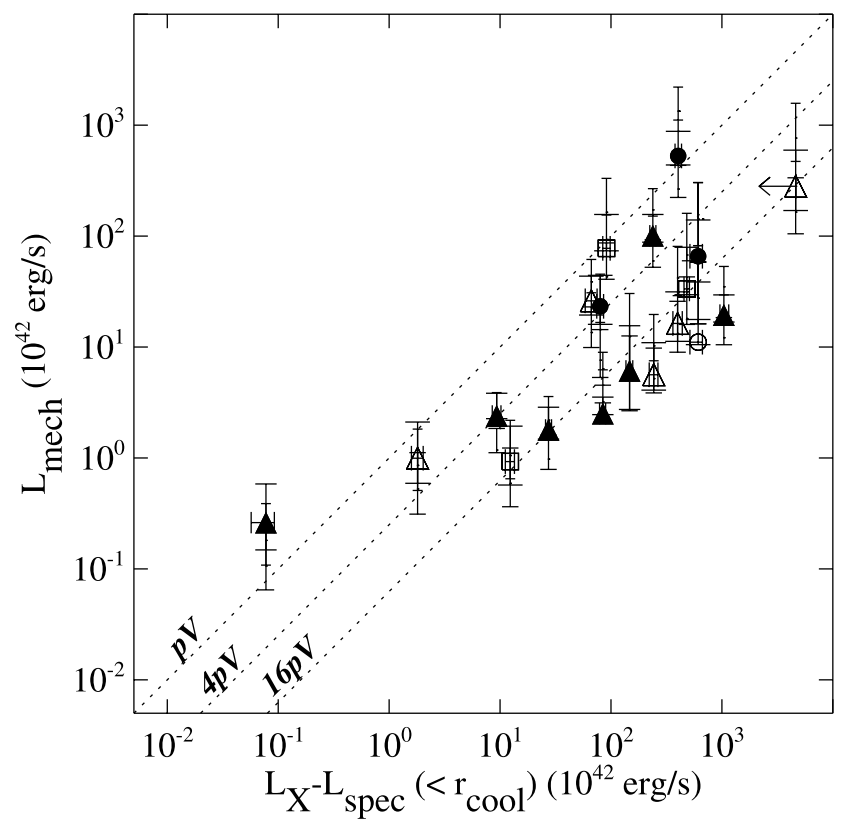

Fig. 2.-Mechanical luminosity vs. total luminosity minus the spectroscopic estimate of the cooling luminosity. Lines denoting $L_{\mathrm{X}}-L_{\mathrm{spec}}=L_{\text {mech }}$ are shown for the assumptions of $p V, 4 p V$, and $16 p V$ energy in the bubbles. Symbols and error bars as in Fig. 1; the arrow denotes an upper limit.

total luminosity of the X-ray-emitting gas from within the cooling radius, as an estimate of the classical, or morphological, cooling luminosity in the absence of heating and $L_{\text {spec }}$, the spectral estimate of the cooling luminosity within the cooling radius, as the luminosity of the gas cooling to low temperatures. The cooling luminosity, $L_{\mathrm{X}}-L_{\mathrm{spec}}$, must be offset by heating in order to prevent the gas from cooling to low temperatures. We note that this quantity ignores non-X-ray cooling, such as ultraviolet and optical emission predicted to result from cooling by thermal conduction inside magnetic flux loops (Soker 2004) or a long reconnected magnetic field lines between cold clouds and the ICM (Soker et al. 2004). Any such emission would lower the cooling luminosity, which must be balanced by heating. Figure 2 shows the mechanical luminosity plotted against $L_{\mathrm{X}}-L_{\text {spec }}$ for our sample. The diagonal lines represent equality between cooling and heating, assuming energy inputs of $p V, 4 p V$, and $16 p V$ per cavity. The data are derived from Tables 2 and 3. For RBS 797, an upper limit is shown. The cooling luminosity for RBS 797 is poorly constrained by the spectrum, which consists of only $\sim 9000$ counts after cleaning. RBS 797, while very luminous, is the most distant cluster in our sample and has the shortest exposure time (see $\S 2$ ).

Figure 2 shows several objects, such as Hydra A, Cygnus A, and M84, whose cavities can contain enough energy to balance radiative losses, at least temporarily, with nearly $1 \mathrm{pV}$ of heat input per cavity. The remaining objects, which require between a few and $\sim 20 p V$ per cavity to balance cooling, would do so with varying degrees of difficulty. As discussed above, $\sim 2 p V$ would be deposited within the cooling radius by an adiabatic bubble containing relativistic plasma. Up to $4 p V$ is available if the cavities are relativistic and nonadiabatic, and there may be further energy input if they are overpressured or produce a shock when they are formed. Therefore, the objects that require $\sim 4 p V$ or less may reasonably be supplied with enough energy in the cavities to balance cooling, depending on the detailed dynamics (the heat also needs to be distributed inside the cooling radius to match the distribution of radiative losses). Provided that the true radio cycling timescale ranges between $t_{c_{s}}$ and $t_{r}$, the cavities in one-quarter to one-half of the objects in our sample contain enough energy to offset radiation losses. This would be true of the cavities in the remaining objects only if they are significantly nonadiabatic, as outlined in $\S 5.2$. Bear in mind that our conclusions depend on the adopted cooling radius (see $\S 3.2$ ), measurement uncertainties in the cavity sizes, and the cavity production timescale. Nevertheless, we can safely conclude that cooling can plausibly be balanced by bubble heating in some, but not all, systems.

It is unnecessary to balance the entire luminosity, $L_{\mathrm{X}}-$ $L_{\text {spec }}$, by bubble heating alone if there are other forms of heating present. A possible source of heating is thermal conduction, which, as demonstrated by Voigt \& Fabian (2004), could supply a significant amount of heat. Using a sample similar to our own, Voigt \& Fabian found that thermal conduction can reduce the cooling luminosity by factors of $\sim 2-3$ in some objects. Although they are difficult to find in X-ray images, shocks associated with the expanding cavities can deposit additional energy into the ICM. Deep Chandra images of a growing number of objects, including Cygnus A (Wilson et al. 2003), NGC 4636 (Jones et al. 2002), M87 (Forman et al. 2004), and Perseus (Fabian et al. 2003a), show surface brightness discontinuities that may be associated with weak shocks. In Cygnus A and M87, the shocks imply that the radio source may provide several times the upper limit of the luminosity seen in the bubbles, under the assumption of $4 p \mathrm{~V}$ of energy per bubble (Wilson et al. 2003; Forman et al. 2004). It may therefore be a combination of heating mechanisms that leads to quenched cooling, as suggested by several authors (Brighenti \& Mathews 2002, 2003; Kim \& Narayan 2003; Ruszkowski \& Begelman 2002).

It is important to note that our sample is biased toward systems with visible evidence of X-ray cavities and does not represent clusters as a whole. Many clusters, including some with large cooling flows, do not contain cavities (e.g., A1068; Wise et al. 2004; McNamara et al. 2004). These objects may have very different reheating histories than the objects discussed here. In this sense, the objects presented here represent the best-case examples for reheating the ICM by energetic bubbles. Our analysis does not imply that all cooling flows can be quenched in this fashion.

\subsection{Trends between X-Ray and Mechanical Luminosities}

Figure 2 shows a trend between the X-ray luminosity and bubble mechanical luminosity, with the sense that systems with larger X-ray luminosities also have larger mechanical luminosities. This trend extends over a dynamic range of $\sim 1000$ in both X-ray and mechanical luminosity. Just such a trend would be expected were the cooling and heating of the ICM coupled in some fashion. Several studies (e.g., Rosner \& Tucker 1989; Binney \& Tabor 1995; David et al. 2001; Quilis et al. 2001; Churazov et al. 2002) have proposed that cooling is balanced by heating in a self-regulated feedback loop. The feedback loop is driven by episodic radio activity fueled by cooling and accretion onto a central black hole. The accretion energy is then returned to the ICM through an AGN outburst, including the action of the radio cavities, which temporarily arrests cooling. At later times, the center of the system settles down and the cooling flow is reestablished. During the cooling cycle, molecular gas (Edge 2001) accumulates and star formation ensues (McNamara \& O'Connell 1989; Johnstone et al. 1987), albeit at substantially lower levels than expected 
in steady-cooling models (Fabian 1994). Even if the radio bubbles are not the main source of heat, Figure 2 suggests that AGN feedback is intimately involved in the process that prevents a large cooling flow from forming.

The apparent correlation in Figure 2 should be treated with caution. As noted above, our sample was selected from clusters in the Chandra archive with fairly obvious cavities in their cores and neglects those without obvious cavities. Other clusters are known to have substantial cooling luminosities commensurate with the observed levels of cold gas and star formation, yet contain no cavities and have low radio power. A prime example is A1068 (Wise et al. 2004; McNamara et al. 2004). Similar objects would appear in the lower right of this diagram, tending to weaken the correlation. On the other hand, it is unlikely that we would have missed objects with powerful cavities, which would lie in the upper part of this diagram. Therefore, the distribution of points may represent an upper envelope in mechanical luminosity as a function of X-ray luminosity. Such a distribution would be consistent with the feedback hypothesis, if objects like A1068 are in an extended cooling phase in which the central galaxies have experienced substantial levels of accretion in the past $100 \mathrm{Myr}$ or so, when the radio source has not had a chance to create cavities capable of reducing or quenching cooling.

We have investigated the degree to which other systematic effects may lead to an unphysical luminosity-luminosity correlation. For example, Elvis et al. (1978) pointed out that a sample of objects with a small range of fluxes and a large range of distances will show a correlation in a luminosity-versusluminosity plot, even if there is no intrinsic correlation in the sample. Our sample, however, has a large range of radio fluxes (from $\sim 10$ to $\sim 10^{6} \mathrm{mJy}$ ). The cavities in our sample also cover a large range of projected angular sizes (from $\sim 3^{\prime \prime}$ to $\sim 35^{\prime \prime}$ ). We believe, then, that these potential effects are unlikely to account entirely for the trends seen in Figures 1 and 2 .

Selection bias may also contribute to the correlations. Small cavities are easily overlooked in distant objects, since cavities of a given linear size become more difficult to detect as their angular sizes decrease with increasing distance. Conversely, in very nearby objects, such as M87, we may miss larger bubbles that lie outside the detector. Furthermore, other considerations, such as the bubble position, affect the detectability (Ensslin \& Heinz 2002). The consequences of these and other effects on our selection function will be addressed in the future using a larger and better-defined sample of clusters, including a more sophisticated approach to placing limits on cavities that may exist in clusters but were missed by the observations.

\subsection{Summary}

We have presented an analysis of 18 systems taken from the Chandra archive having clear evidence of cavities in their $\mathrm{X}$-ray emission. We find that the energy associated with the cavities is sufficient to substantially reduce or quench cooling in nearly half of the objects in our sample. However, this mechanism alone probably does not provide a general solution to the cooling problem, unless X-ray cavities probe only a small fraction of the total kinetic luminosity of radio sources. In addition, we have discovered a trend between the cooling X-ray luminosity and the mechanical energy of the cavities, with the sense that more luminous systems produce larger and more energetic cavities. The trend, or envelope, may have been established by a self-regulated cooling and feedback mechanism acting in many systems. The existence of such a mechanism in relatively nearby clusters, where the detailed physics can be examined, may provide significant insight on the process of galaxy formation that prevails at large redshifts (e.g., Voit \& Ponman 2003). A similar mechanism may regulate the growth of galaxy halos during the dissipative stages of their development (Dubinski 1994) and may be an agent responsible for the detailed correlation between black hole mass and velocity dispersion of spheroids (Fabian et al. 2002b). We have measured for the first time the distribution of the ratio of kinetic luminosity to monochromatic radio luminosity for a sample of radio sources. The ratio varies widely, with most objects ranging between few and a few hundred, assuming $1 p V$ of energy per cavity. X-ray cavities provide a unique probe of the mechanical power of radio jets, independently of the radio properties themselves.

Our future plans include expanding the sample size and acquiring better and more uniform radio data. In addition, we plan to extend our understanding of the detectability function of bubbles, using simulations of images with a wide range of exposure and signal-to-noise ratios.

We acknowledge helpful discussions with Mangala Sharma, Liz Blanton, Frazer Owen, Hui Li, Phil Kronberg, and David De Young, and we thank Carlo Nipoti for pointing out an error in the Centaurus radio flux in an earlier version. This work was supported by Long Term Space Astrophysics Grant NAG5-11025, Chandra Archive Grant AR2-3007X, and a grant from the Department of Energy through the Los Alamos National Laboratory.
Basson, J. F., \& Alexander, P. 2003, MNRAS, 339, 353

Becker, R. H., White, R. L., \& Edwards, A. L. 1991, ApJS, 75, 1

Bender, R., Saglia, R. P., \& Gerhard, O. E. 1994, MNRAS, 269, 785

Bertschinger, E., \& Meiksin, A. 1986, ApJ, 306, L1

Bicknell, G. V., Dopita, M. A., \& O'Dea, C. P. 1997, ApJ, 485, 112

Binney, J. 2004, in The Riddle of Cooling Flows in Galaxies and Clusters of

Galaxies, ed. T. H. Reiprich, J. C. Kempner, \& N. Soker (Charlottesville:

Univ. Virginia), http://www.astro.virginia.edu/coolflow

Binney, J., \& Tabor, G. 1995, MNRAS, 276, 663

Binney, J., \& Tremaine, S. 1987, Galactic Dynamics (Princeton: Princeton Univ. Press)

Blakeslee, J. P., \& Tonry, J. L. 1992, AJ, 103, 1457

Blanton, E. L., Sarazin, C. L., \& McNamara, B. R. 2003, ApJ, 585, 227

Blanton, E. L., Sarazin, C. L., McNamara, B. R., \& Wise, M. W. 2001, ApJ, $558, \mathrm{~L} 15$

Böhringer, H., \& Hensler, G. 1989, A\&A, 215, 147

Böhringer, H., Matsushita, K., Churazov, E., Ikebe, Y., \& Chen, Y. 2002, A\&A, 382,804

\section{REFERENCES}

Böhringer, H., \& Morfill, G. E. 1988, ApJ, 330, 609

Böhringer, H., Voges, W., Fabian, A. C., Edge, A. C., \& Neumann, D. M. 1993, MNRAS, 264, L25

Bregman, J. N., \& David, L. P. 1988, ApJ, 326, 639

Brighenti, F., \& Mathews, W. G. 2002, ApJ, 573, 542 . 2003, ApJ, 587, 580

Brüggen, M. 2002, ApJ, 571, L13

2003, ApJ, 592, 839

Brüggen, M., \& Kaiser, C. R. 2001, MNRAS, 325, 676

Brüggen, M., Kaiser, C. R., Churazov, E., \& Ensslin, T. A. 2002, MNRAS, 331,545

Burbidge, G., \& Crowne, A. H. 1979, ApJS, 40, 583

Carilli, C. L., Perley, R. A., \& Harris, D. E. 1994, MNRAS, 270, 173

Carollo, C. M., Danzinger, I. J., \& Buson, L. 1993, MNRAS, 265, 553

Carter, D., Inglis, I., Ellis, R. S., Efstathiou, G., \& Godwin, J. G. 1985, MNRAS, 212, 471

Churazov, E., Brüggen, M., Kaiser, C. R., Böhringer, H., \& Forman, W. R. 2001, ApJ, 554, 261 
Churazov, E., Sunyaev, R., Forman, W., \& Böhringer, H. 2002, MNRAS, 332, 729

Ciotti, L., \& Ostriker, J. P. 2001, ApJ, 551, 131

Condon, J. J., Cotton, W. D., Greisen, E. W., Yin, Q. F., Perley, R. A., Taylor, G. B., \& Broderick, J. J. 1998, AJ, 115, 1693

David, L. P., Nulsen, P. E. J., McNamara, B. R., Forman, W., Jones, C.,

Ponman, T., Robertson, B., \& Wise, M. 2001, ApJ, 557, 546

De Young, D. S. 1993, ApJ, 405, L13

. 2003, MNRAS, 343, 719

Dolag, K., Jubelgas, M., Springel, V., Borgani, S., \& Rasia, E. 2004, ApJL, in press

Dubinski, J. 1994, ApJ, 431, 617

Edge, A. C. 2001, MNRAS, 328, 762

Elvis, M., Maccacaro, T., Wilson, A. S., Ward, M. J., Penston, M. V., Fosbury, R. A. E., \& Perola, G. C. 1978, MNRAS, 183, 129

Ensslin, T. A., \& Heinz, S. 2002, A\&A, 384, L27

Ettori, S., Fabian, A. C., Allen, S. W., \& Johnstone, R. M. 2002, MNRAS, 331,635

Fabian, A. C. 1994, ARA\&A, 32, 277

Fabian, A. C., Celotti, A., Blundell, K. M., Kassim, N. E., \& Perley, R. A. 2002a, MNRAS, 331, 369

Fabian, A. C., Mushotzky, R. F., Nulsen, P. E. J., \& Peterson, J. R. 2001, MNRAS, 321, L20

Fabian, A. C., Sanders, J. S., Allen, S. W., Crawford, C. S., Iwasawa, K., Johnstone, R. M., Schmidt, R. W., \& Taylor, G. B. 2003a, MNRAS, 344, L43

Fabian, A. C., Sanders, J. S., Crawford, C. S., Conselice, C. J., Gallagher, J. S., III, \& Wyse, R. F. G. 2003b, MNRAS, 344, L48

Fabian, A. C., Wilman, R. J., \& Crawford, C. S. 2002b, MNRAS, 329, L8

Fabian, A. C., et al. 2000, MNRAS, 318, L65

Finoguenov, A., \& Jones, C. 2001, ApJ, 547, L107

Fisher, D., Illingworth, G., \& Franx, M. 1995, ApJ, 438, 539

Forman, W., et al. 2004, ApJ, submitted (astro-ph/0312576)

Fujita, Y., Sarazin, C. L., Kempner, J. C., Rudnick, L., Slee, O. B., Roy, A. L., Andernach, H., \& Ehle, M. 2002, ApJ, 575, 764

Gull, S. F., \& Northover, K. J. E. 1973, Nature, 244, 80

Heckman, T. M., Illingworth, G. D., Miley, G. K., \& van Breugel, W. J. M. 1985, ApJ, 299, 41

Heinz, S., Choi, Y.-Y., Reynolds, C. S., \& Begelman, M. C. 2002, ApJ, 569, L79

Heinz, S., Reynolds, C. S., \& Begelman, M. C. 1998, ApJ, 501, 126

Johnstone, R. M., Allen, S. W., Fabian, A. C., \& Sanders, J. S. 2002, MNRAS, 336,299

Johnstone, R. M., Fabian, A. C., \& Nulsen, P. E. J. 1987, MNRAS, 224, 75

Jones, C., Forman, W., Vikhlinin, A., Markevitch, M., David, L., Warmflash, A., Murray, S., \& Nulsen, P. E. J. 2002, ApJ, 567, L115

Kaastra, J. S., et al. 2004, A\&A, 413, 415

Kaiser, C. R., \& Binney, J. 2003, MNRAS, 338, 837

Kim, W.-T., \& Narayan, R. 2003, ApJ, 596, 889

Kühr, H., Witzel, A., Pauliny-Toth, I. I. K., \& Nauber, U. 1981, A\&AS, 45, 367

Lane, W. M., Clarke, T. E., Taylor, G. B., Perley, R. A., \& Kassim, N. E. 2004, AJ, 127, 48

Loewenstein, M., Zweibel, E. G., \& Begelman, M. C. 1991, ApJ, 377, 392

Makishima, K., et al. 2001, PASJ, 53, 401

Mathews, W. G., Brighenti, F., Buote, D. A., \& Lewis, A. D. 2003, ApJ, 596,159

Mazzotta, P., Edge, A. C., \& Markevitch, M. 2003, ApJ, 596, 190

Mazzotta, P., Kaastra, J. S., Paerels, F. B., Ferrigno, C., Colafrancesco, S., Mewe, R., \& Forman, W. R. 2002, ApJ, 567, L37

McNamara, B. R., \& O’Connell, R. W. 1989, AJ, 98, 2018

McNamara, B. R., Wise, M. W., \& Murray, S. S. 2004, ApJ, 601, 173

McNamara, B. R., et al. 2000, ApJ, 534, L135 2001, ApJ, 562, L149

Molendi, S., \& Pizzolato, F. 2001, ApJ, 560, 194

Nulsen, P. E. J., David, L. P., McNamara, B. R., Jones, C., Forman, W. R., \& Wise, M. 2002, ApJ, 568, 163
Omma, H., Binney, J., Bryan, G., \& Slyz, A. 2004, MNRAS, 348, 1105

Pedlar, A., Ghataure, H. S., Davies, R. D., Harrison, B. A., Perley, R., Crane, P. C., \& Unger, S. W. 1990, MNRAS, 246, 477

Peterson, J. R., Kahn, S. M., Paerels, F. B., Kaastra, J. S., Tamura, T., Bleeker, J. A. M., Ferrigno, C., \& Jernigan, J. G. 2003, ApJ, 590, 207

Peterson, J. R., et al. 2001, A\&A, 365, L104

Quilis, V., Bower, R. G., \& Balogh, M. L. 2001, MNRAS, 328, 1091

Reynolds, C. S., Heinz, S., \& Begelman, M. C. 2002, MNRAS, 332, 271

Robinson, K., et al. 2004, ApJ, 601, 621

Rosner, R., \& Tucker, W. H. 1989, ApJ, 338, 761

Ruszkowski, M., \& Begelman, M. C. 2002, ApJ, 581, 223

Sanders, J. S., \& Fabian, A. C. 2002, MNRAS, 331, 273

Sarazin, C. L., Baum, S. A., \& O’Dea, C. P. 1995a, ApJ, 451, 125

Sarazin, C. L., Burns, J. O., Roettiger, K., \& McNamara, B. R. 1995b, ApJ, 447,559

Schindler, S., Castillo-Morales, A., De Filippis, E., Schwope, A., \& Wambsganss, J. 2001, A\&A, 376, L27

Schmidt, R. W., Fabian, A. C., \& Sanders, J. S. 2002, MNRAS, 337, 71

Slee, O. B. 1995, Australian J. Phys., 48, 143

Slee, O. B., Roy, A. L., Murgia, M., Andernach, H., \& Ehle, M. 2001, AJ, 122,1172

Slee, O. B., \& Siegman, B. C. 1988, MNRAS, 235, 1313

Smith, D. A., Wilson, A. S., Arnaud, K. A., Terashima, Y., \& Young, A. J. 2002, ApJ, 565, 195

Smith, E. P., Heckman, T. M., \& Illingworth, G. D. 1990, ApJ, 356, 399

Soker, N. 2003, MNRAS, 342, 463 2004, MNRAS, in press (astro-ph/0311014)

Soker, N., Blanton, E. L., \& Sarazin, C. L. 2004, in The Riddle of Cooling Flows in Galaxies and Clusters of Galaxies, ed. T. H. Reiprich, J. C. Kempner, \& N. Soker (Charlottesville: Univ. Virginia), http://www.astro .virginia.edu/coolflow

Soker, N., White, R. E., III, David, L. P., \& McNamara, B. R. 2001, ApJ, 549,832

Spinrad, H., Djorgovski, S., Marr, J., \& Aguilar, L. 1985, PASP, 97, 932

Sun, M., Jones, C., Murray, S. S., Allen, S. W., Fabian, A. C., \& Edge, A. C. 2003, ApJ, 587, 619

Tabor, G., \& Binney, J. 1993, MNRAS, 263, 323

Tamura, T., et al. 2001, A\&A, 365, L87

Taylor, G. B., Barton, E. J., \& Ge, J. 1994, AJ, 107, 1942

Tonry, J. L. 1985, AJ, 90, 2431

Tucker, W. H., \& David, L. P. 1997, ApJ, 484, 602

Tucker, W. H., \& Rosner, R. 1983, ApJ, 267, 547

Voigt, L. M., \& Fabian, A. C. 2004, MNRAS, 347, 1130

Voigt, L. M., Schmidt, R. W., Fabian, A. C., Allen, S. W., \& Johnstone, R. M. 2002, MNRAS, 335, L7

Voit, G. M., \& Ponman, T. J. 2003, ApJ, 594, L75

von Hoerner, S. 1974, in Galactic and Extragalactic Radio Astronomy, ed. G. L. Verschuur \& K. I. Kellermann (Berlin: Springer), 353

Vrtilek, J. M, Grego, L., David, L. P., Ponman, T. J., Forman, W., Jones, C., \& Harris, D. E. 2002, APS Meeting, B17.107, http://www.aps.org/meet/ APR02/baps/abs/S200107.html

Wilson, A. S., Young, A. J., \& Smith, D. A. 2003, in ASP Conf. Ser. 290, Active Galactic Nuclei: From Central Engine to Host Galaxy, ed. S. Collin, F. Combes, \& I. Shlosman (San Francisco: ASP), 141

Wise, M. W., McNamara, B. R., \& Murray, S. S. 2004, ApJ, 601, 184

Wright, A. E., Griffith, M. R., Burke, B. F., \& Ekers, R. D. 1994, ApJS, 91, 111

Wright, A. E., Griffith, M. R., Hunt, A. J., Troup, E., Burke, B. F., \& Ekers, R. D. 1996, ApJS, 103, 145

Wright, A. E., \& Otrupcek, R. 1990, Parkes Radio Sources Catalogue (Epping: ATNF)

Young, A. J., Wilson, A. S., \& Mundell, C. G. 2002, ApJ, 579, 560

Zakamska, N. L., \& Narayan, R. 2003, ApJ, 582, 162 